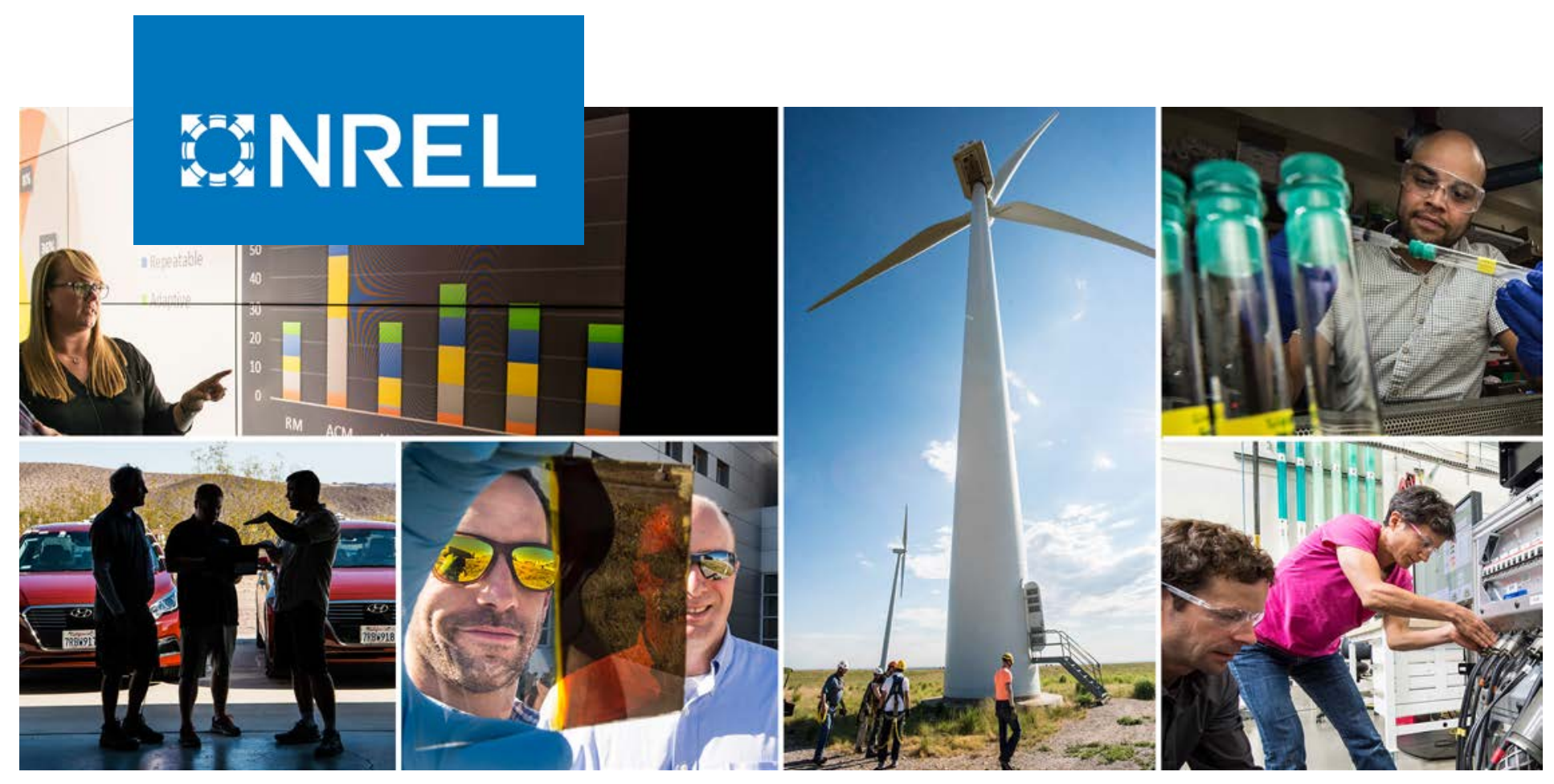

\title{
Impact of Aggregated PV on Subsynchronous Torsional Interaction
}

\section{Preprint}

Rasel Mahmud, Andy Hoke, and Jonathan White

National Renewable Energy Laboratory

Presented at the 47th IEEE Photovoltaic Specialists Conference (PVSC 47) June 15-August 21, 2020

NREL is a national laboratory of the U.S. Department of Energy

Office of Energy Efficiency \& Renewable Energy

Operated by the Alliance for Sustainable Energy, LLC

This report is available at no cost from the National Renewable Energy Laboratory (NREL) at www.nrel.gov/publications.

\section{Conference Paper}

NREL/CP-5D00-74790

July 2020 


\title{
GNREL
}

\section{Impact of Aggregated PV on Subsynchronous Torsional Interaction}

\section{Preprint}

\author{
Rasel Mahmud, Andy Hoke, and Jonathan White
}

National Renewable Energy Laboratory

\section{Suggested Citation}

Mahmud, Rasel, Andy Hoke, and Jonathan White. 2020. Impact of Aggregated PV on

Subsynchronous Torsional Interaction: Preprint. Golden, CO: National Renewable Energy Laboratory. NREL/CP-5D00-74790. https://www.nrel.gov/docs/fy20osti/74790.pdf.

NREL is a national laboratory of the U.S. Department of Energy Office of Energy Efficiency \& Renewable Energy Operated by the Alliance for Sustainable Energy, LLC

This report is available at no cost from the National Renewable Energy Laboratory (NREL) at www.nrel.gov/publications.

Contract No. DE-AC36-08GO28308
Conference Paper NREL/CP-5D00-74790 July 2020

National Renewable Energy Laboratory 15013 Denver West Parkway Golden, CO 80401 303-275-3000 • www.nrel.gov 


\section{NOTICE}

This work was authored by the National Renewable Energy Laboratory, operated by Alliance for Sustainable Energy, LLC, for the U.S. Department of Energy (DOE) under Contract No. DE-AC36-08GO28308. Funding provided by U.S. Department of Energy Office of Energy Efficiency and Renewable Energy (EERE) Solar Energy Technologies Office and Office of Cybersecurity, Energy Security, and Emergency Response. The views expressed herein do not necessarily represent the views of the DOE or the U.S. Government.

This report is available at no cost from the National Renewable Energy Laboratory (NREL) at www.nrel.gov/publications.

U.S. Department of Energy (DOE) reports produced after 1991 and a growing number of pre-1991 documents are available free via www.OSTI.gov.

Cover Photos by Dennis Schroeder: (clockwise, left to right) NREL 51934, NREL 45897, NREL 42160, NREL 45891, NREL 48097, NREL 46526.

NREL prints on paper that contains recycled content. 


\section{Impact of Aggregated PV on Subsynchronous Torsional Interaction}

\author{
Rasel Mahmud \\ National Renewable Energy Laboratory \\ Golden \\ Colorado 80401 \\ rasel.mahmud@nrel.gov
}

\author{
Andy Hoke \\ National Renewable Energy Laboratory \\ Golden \\ Colorado 80401 \\ andy.hoke@nrel.gov
}

\author{
Jonathan White \\ National Renewable Energy Laboratory \\ Golden \\ Colorado 80401 \\ jonathan.white@nrel.gov
}

\begin{abstract}
This paper investigates induced oscillation (specifically subsynchronous torsional interactions) in power systems arising from photovoltaic (PV) power modulation. Time domain simulations of a modified IEEE first-benchmark system are presented and analyzed. In the IEEE benchmark system, a PV generator was added at the same bus where the synchronous generator is connected. The impact of different parameters of the PV inverters' volt-volt-ampere reactive (volt-var) curve on SSTI is examined. The most severe impact found arises from the time response of the inverter's reactive power change. A second-order time response with natural frequency matching the rotor speed deviation oscillation frequency and low-damping ratio quickly drives the system to an unstable region. Although it is highly unlikely that conditions necessary for distributed energy resource-induced oscillations would occur in the field, with knowledge of this vulnerability in mind, inverters' control parameters can be designed to avoid it.
\end{abstract}

\section{INTRODUCTION}

The impact of inverter-based resources on power system stability has recently gained attention [1], [2] as the percentage of inverter-based renewable generation is increasing rapidly [3]. Evaluations of voltage source converters used in inverter-based resource and their circuit parameters, control strategies, and control parameters for power system stability analysis have been addressed in several publications [4]. Most published literature dealing with inverter-based resources and power system stability have mainly considered wind power plant and grid stability issues. Similarly, high-voltage DC [5] and flexible AC transmission systems [6] have also been studied extensively. There exists a gap, however, in studies related to power system stability in the presence of photovoltaics (PV). The reason for this could be comparatively less penetration of PV than other energy sources as well as the location of PV connections, which are mainly on distribution circuits. PV penetration is also increasing very fast [7], so the impact of aggregated PV on power system stability can no longer be ignored. This situation could be more critical when instantaneous energy supplied by PV approaches $50 \%$ of total energy supplied by the grid in some locations.

It is known that inverter-based resources under certain conditions can provide negative damping to a subsynchronous resonance (SSR) mode, of which subsynchronous torsional interaction (SSTI) is a subset, thus increasing oscillatory behavior [8], [9], [10]. In an SSTI, torsional modes of the generator and turbine masses and shafts oscillate with external drivers such as series-connected line-compensation capacitors or power electronic devices. This can push the grid into a critically unstable state. In this paper, we investigate whether grid support functions of aggregated PV inverters impact SSTI. In that regard, we consider SSTI mitigation techniques using inverter-based resources and explore potential impacts of voltvar function of PV on SSTI.

\section{Model Background}

A modified IEEE first-benchmark system [11], as shown in Fig. 1, can be used to verify the effectiveness of aggregated PV in mitigating SSTI. The steam turbine model of the synchronous generator has a two-stage steam turbine consisting of a shaft connecting two masses: low pressure and high pressure. The shaft is coupled with the synchronous machine by a second shaft, for a total of three masses. The IEEE benchmark system was modified by adding a PV generator at the same bus where the synchronous generator is connected. The PV generator can be taken to represent a single large PV plant or an aggregation of many distributed PV plants. The synchronous generator is rated for $600 \mathrm{MVA}$, and the aggregated rating of the PV is 300 MVA. The synchronous generator and the aggregated PV are connected to the rest of the network via a transformer and a series-compensated transmission line. This very simple model does not represent any specific real system, so the simulations presented here should be considered preliminary. An electromagnetic transient model of this benchmark system was developed in MATLAB/Simulink. The rotor speed oscillates around its steady-state value when there is an SSTI. These oscillations can be triggered by a disturbance, such as a fault in the transmission line, especially in a series-compensated line [12].

\section{DAmping SSTI with AggRegated PV}

R. K. Verma et al [13] showed that a large-scale PV plant can be used as a PV static synchronous compensator to mitigate SSR. Building on that work, we demonstrate that the voltage regulation feature of smart inverters could possibly impact SSTI if configured improperly. The approach proposed by [13] uses the generator rotor speed deviation from nominal 


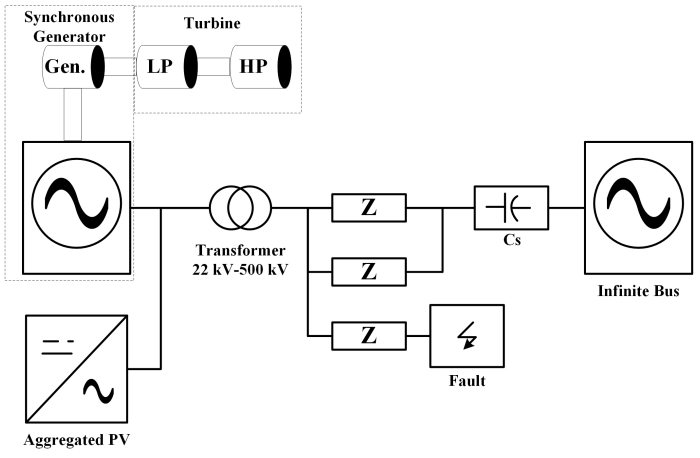

Fig. 1. Modified IEEE first-benchmark model for testing SSTI induced by aggregated PV

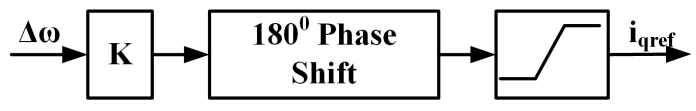

Fig. 2. SSTI damping controller

$(\Delta \omega)$ to generate a reference reactive current command $\left(i_{\text {qref }}\right)$ to nullify the rotor speed deviation, thus mitigating the SSTI. Fig. 2 shows the SSTI damping controller as proposed by [13]. A simulation test was performed to verify that aggregated PV can suppress SSTI when a fault in the transmission line triggers it. Fig. 3 shows the simulation result. At $t=0.022 \mathrm{~s}$, an SSTI event is initiated because of a fault in the transmission line. The reactive current from the aggregated PV is controlled by the damping controller shown in Fig. 2. Within $2 \mathrm{~s}$, the rotor speed deviation is completely damped because of the reactive current injection from the aggregated inverter.

\section{IMPACT OF VOLT-VAR CONTROL ON SSTI}

In this example, the aggregated PV uses the information of rotor speed deviation in real time to modulate the inverter reactive power. In practice, obtaining real-time information on rotor speed deviation could be very difficult. Nonetheless, it is evident that the reactive power output of aggregated PV has a significant impact on the stability of the synchronous generator. We, therefore, investigate the effects of other PV reactive power modulation techniques on the SSTI. A widely adopted method for PV reactive power regulation is volt-var control, in which reactive power is modulated in response to local voltage measurements. We examine different parameters of volt-var curves and their impact on the stability of the synchronous generator. Fig. 4(a) shows a regular volt-var curve that can be implemented in most smart inverters. The parameters of the volt-var curves can be changed to have altered forms of volt-var curves, as shown in Fig. 4(b) and Fig. 4(c). The impact of horizontal shifts of the volt-var curve deadband along the voltage axis and vertical shifts of the voltvar deadband along the VAR axis are examined. The recently published IEEE 1547-2018 [14] standard specifies a range of allowable settings for different volt-var curve parameters. By changing the volt-var curve parameters within the allowable

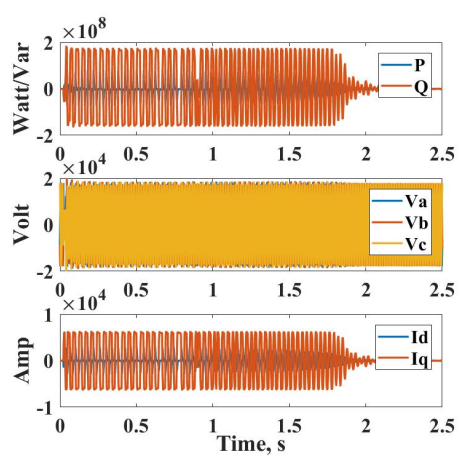

(a)
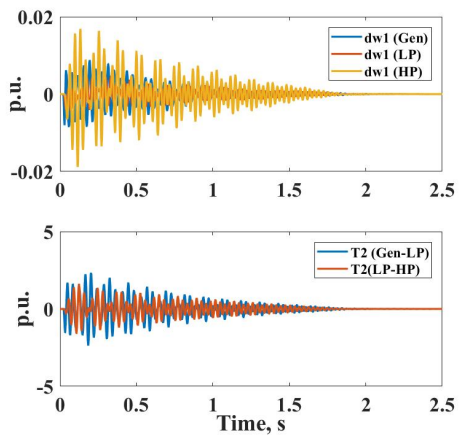

(b)

Fig. 3. SSTI mitigation using aggregated PV: (a) power, voltage, and current measurements of aggregated PV output; and (b) synchronous generator response; the top curve shows the rotor speed deviations of different masses of the synchronous generator, and the lower plot shows the torques, in p.u., transmitted by masses of the generator, low-pressure, and high-pressure side of the turbine.

range, the altered forms of the volt-var curves shown in Fig. 4 can be achieved. The impact of the altered volt-var curves on SSTI is measured by the maximum deviation of the synchronous generator rotor speed from nominal. Fig. 5(a) shows the baseline case when there is no reactive power generation or absorption by the aggregated PV. A fault in the transmission line triggers an SSTI event, as evident in Fig. 5(b). Any impact from reactive power modulation by aggregated PV will result in a change in rotor speed deviation. Thus, the maximum rotor speed deviation from nominal will be a good indicator to assess the impact. To complete all the simulations in a reasonable duration, each simulation is run for a 5-s window, unless some other stopping criterion is met. (Details of the stopping criteria are discussed later.) Fig. 5(b) shows that rotor speed deviation continues to grow in the baseline case, indicating the potential for SSTI exists without $\mathrm{PV}$. The maximum rotor speed deviation metric used in this paper represents the maximum rotor speed deviation reached during the 5-s window, or in a shorter duration window when the simulation is stopped sooner. Larger rotor speed deviations represent an increased magnitude of SSTI. 


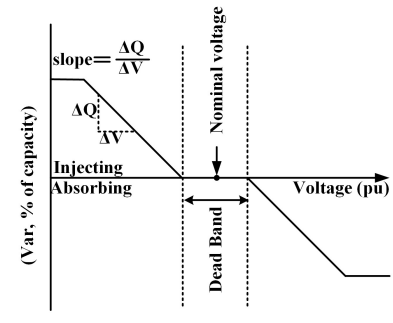

(a)

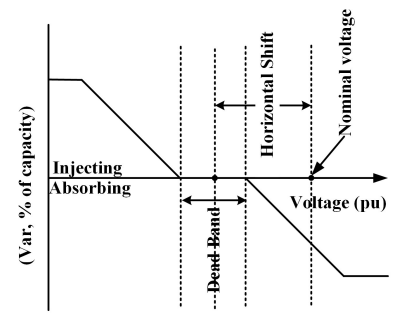

(b)

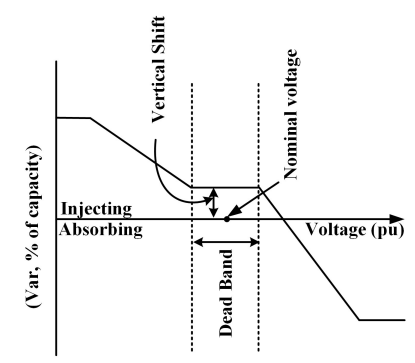

(c)

Fig. 4. Different volt-var curves: (a) regular volt-var curve, (b) horizontal shift of deadband from nominal voltage along the voltage axis, and (c) vertical shift of deadband along the var axis

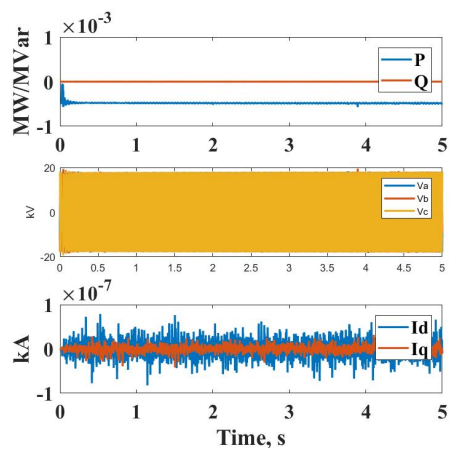

(a)
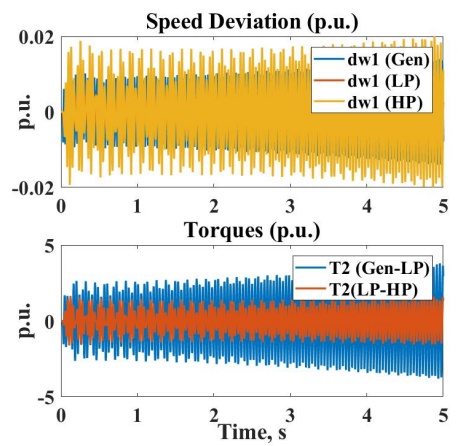

(b)

Fig. 5. Baseline case when the aggregated PV is not generating or absorbing any power: (a) power, voltage and current measurements of aggregated PV output and (b) synchronous generator response

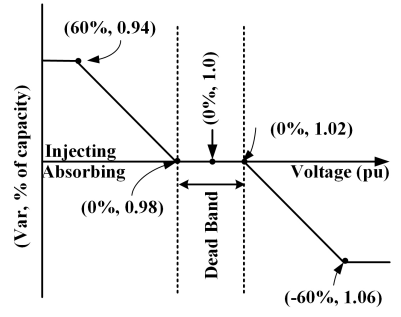

Fig. 6. Baseline volt-var curve

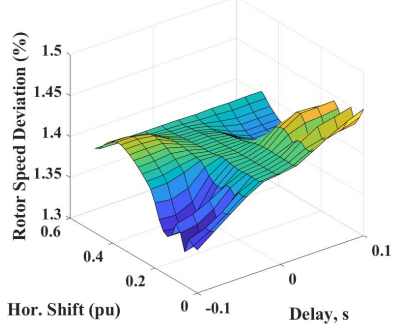

Fig. 7. Impact of shifting volt-var curve horizontally and changing volt-var controller delay time on rotor speed deviation

\section{A. Baseline Volt-var Modulation}

Various simulation tests are conducted to investigate the sensitivity of the SSTI to volt-var control parameters. Fig. 6 shows the baseline volt-var curve used for the simulations. In these tests, the PV output is controlled based on only local information (i.e., without using the rotor speed information). The PV generator voltage-trip settings are not implemented, allowing testing across a wider range of voltage settings.

\section{B. Worst-Case Analysis: Horizontal Volt-var Shifting}

For a first set of tests, the middle point of the volt-var deadband is horizontally shifted in 0.02-p.u. increments from 0.1 p.u. below nominal voltage to 0.1 p.u. above nominal voltage. The middle point of the deadband is initially set to 0.9 p.u. voltage, then it is incrementally moved toward 1.1 p.u. voltage in 0.02 -p.u. voltage steps. This process is repeated for different values of delays in the volt-var control response, from $0.0002 \mathrm{~s}$ to $0.4802 \mathrm{~s}$, with 0.02 -s incremental steps. Fig. 7 shows the maximum rotor speed deviation for each test in this set. A total of 275 simulations are performed for this set of tests. It is found that shifting the volt-var curve 0.1 p.u. above nominal voltage and using the lowest delay produces maximum rotor speed deviation, although the overall differences in maximum rotor speed deviation are not particularly significant.

\section{Worst-Case Analysis: Vertical Volt-var Shifting}

The goal of the simulation is to find the worst condition that produces maximum rotor speed deviation. The volt-var set points that produced the greatest rotor speed deviation in the previous step are used in subsequent tests. Starting from those settings, the volt-var curve is shifted vertically to see 


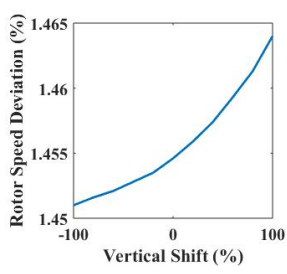

Fig. 8. Impact of shifting volt-var vertically on rotor speed deviation

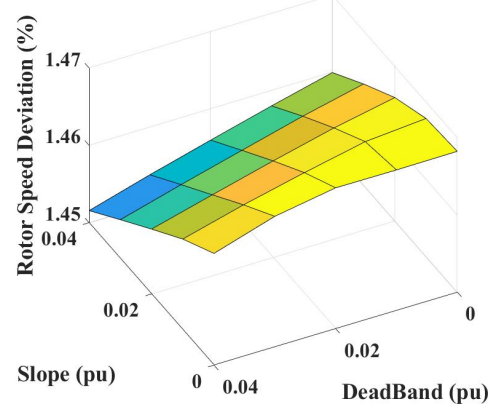

Fig. 9. Impact of change of volt-var Deadband and Steepness on rotor speed deviation

the impact on rotor speed deviation. In this case, the vertical shift happens only on the deadband; other points of the curve are kept constant. Fig. 8 shows a summary of the simulation results. Vertical shifting of the deadband above the baseline position yields maximum rotor speed deviation.

\section{Worst-Case Analysis: Deadband and Steepness of Volt-var Curve}

Once the worst position of the vertically and horizontally shifted volt-var curve is found, simulations are conducted to find how the deadband width and the steepness of the volt-var curve slope impact the rotor speed deviation. Fig. 9 shows a summary of the simulation results. Results show that a steeper slope generally produces more rotor speed deviation. Deadband width is found to have a less significant impact on the rotor speed deviation.

Examination of Fig. 7 through Fig. 9 show that although different values of the parameters defining the volt-var curve have different impacts on the rotor speed deviation, the maximum rotor speed deviation does not greatly change for any of these settings.

\section{Impact of Volt-Var Time Response}

Further investigation of the issue includes consideration of the time response of the volt-var controller. It was previously observed by the authors that the volt-var time-domain dynamics of most commercially available inverters can be modeled with reasonable accuracy using one of three types of time responses: first-order responses, second-order responses, and ramp rate-limited responses [15]. A series of simulation tests is performed on the aggregated inverter to find the impact of

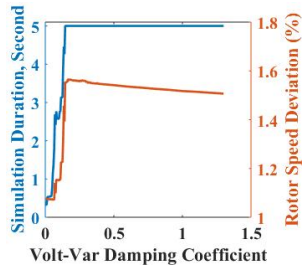

Fig. 10. Impact of damping coefficient in the second-order time response on rotor angle stability

different second-order volt-var responses on the rotor speed deviation; the second-order response can be defined as (1):

$$
H(s)=\frac{\omega_{n}^{2}}{s^{2}+\zeta \omega_{n} s+\omega_{n}^{2}}
$$

where $\omega_{n}$, and $\zeta$ are the natural frequency and damping coefficient of the response, respectively. The previously found worst-case volt-var settings are used while running the tests with different time response parameters. The simulations find that if the natural frequency of the second-order time response matches the frequency of the rotor speed deviation, the resulting SSTI could cause the synchronous machine to become unstable. Whether the SSTI becomes unstable depends on the damping coefficient for the time response and the capacity of the aggregated inverter. For the setup used in these simulations, the frequency of the rotor speed deviation was found to be around $25 \mathrm{~Hz}$. A summary of the simulation results for varying damping coefficients on the rotor angle stability is presented in Fig. 10. All the simulations are set to run for $5 \mathrm{~s}$; however, a stop criterion was set in the simulation to check for rotor speed deviation and output power from the aggregated inverter. If the rotor speed deviates more than $2 \%$ or the aggregated PV reactive power output exceeds $150 \%$ of rated power, the simulation stops, indicating an unstable condition. Fig. 10 shows that the simulation setup quickly reaches instability for damping coefficients in the range of $0-0.17$. Also note that IEEE 1547-2018[14] requires distributed energy resources (DERs) to have adjustable response times. Though a damping coefficient might not be a directly adjustable parameter, a very fast response time might set the value of the damping coefficient within the range that triggers instability. Fig. 11 shows the response of the aggregated PV and synchronous machine rotor speed when the aggregated PV has a secondorder time response with natural frequency matching the rotor speed deviation frequency $(25 \mathrm{~Hz})$ and damping coefficient of 0.005 . Under this condition, the system becomes highly unstable and the aggregated PV output shows reactive power oscillation. The rotor speed deviation also shows increased deviation. Note that such a low damping ratio is unlikely to be found in a realistic inverter.

\section{CONCLUSION}

Time domain simulations were performed to investigate induced oscillation arising from aggregated PV response. A simple IEEE benchmark model with a synchronous generator 


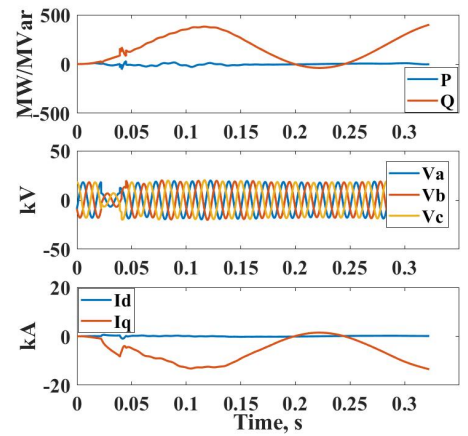

(a)
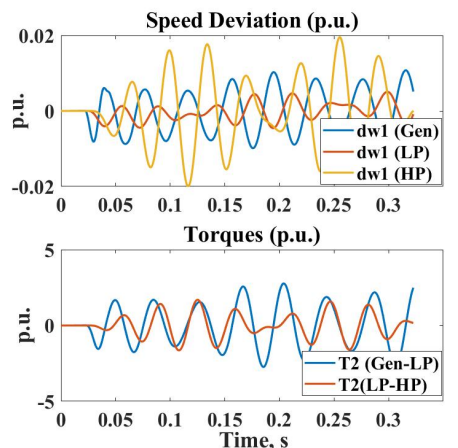

(b)

Fig. 11. Simulation result when the natural frequency of aggregated volt-var response matches rotor speed deviation frequency $(25.12 \mathrm{~Hz})$ with a volt-var damping coefficient of 0.005

was chosen and aggregated PV was added to investigate the induced oscillation phenomena. It was demonstrated that if generator rotor speed information is available in real time, SSTI can be damped by modulating the reactive power of aggregated PV. Considering that obtaining real-time rotor speed data could be very difficult, the effect of altered PV volt-var curves on SSTI was investigated. A large number of electromagnetic transient simulations were performed in MATLAB/Simulink to investigate the induced oscillation. The following observations were made:

- Different parameters of volt-var curves have different levels of impact on SSTI;

- The most severe impact found so far arises from the time response of reactive power change; a second-order time response with natural frequency matching the rotor speed deviation oscillation frequency and low damping ratio drives the system quickly to an unstable region.

Although this research identified a potential impact of aggregated PV on the stability of the electric grid, especially on the ability of aggregated PV to induce oscillations in the power system, the work was limited in scope, and findings should be considered preliminary. The models used were simplified, and the effects of many model parameters were not investigated. Numerous aspects should be investigated further:

- Developing an analytic (mathematical) understanding of the effects of advanced inverter functions on induced oscillations, including SSTI.

- The simplified model used here did not consider SSTI mitigation techniques commonly deployed by synchronous machines. Additionally, PV generator voltage trip settings were not implemented. Further investigation is required to find the impact of such constraints and protections on SSTI induced by aggregated PV.

- A very simple network model (IEEE first-benchmark model) was considered here with series compensation. A more realistic model should be considered in future investigations. The effects of changing the value of the series capacitor or eliminating the capacitor should be investigated. Additionally, the effect of varying the impedance between the synchronous generator and the DER should be investigated.

- A single aggregated PV generator was considered here; in reality, PV systems are often highly distributed. Further investigation is required to find the impact of distributed inverter-based resources on SSTI.

- This research was focused on SSTI, but other modes of oscillations should be investigated.

While it is possible to create the conditions for inverter-based resources to contribute to SSTI in simulation, it is unlikely that such conditions would exist in the field, and such conditions can be avoided through proper selection of inverter control parameters.

\section{ACKNOWLEDGMENT}

This work was supported by the U.S. Department of Energy under Contract No. DE-AC36-08GO28308 with Alliance for Sustainable Energy, LLC, the Manager and Operator of the National Renewable Energy Laboratory. Funding provided by U.S. Department of Energy Office of Energy Efficiency and Renewable Energy (EERE) Solar Energy Technologies Office. The authors acknowledge Dr. Jin Tan of the National Renewable Energy Laboratory for her invaluable assistance and inputs.

\section{REFERENCES}

[1] K. M. Alawasa, Y. A.-R. I. Mohamed, and W. Xu, "Modeling, Analysis, and Suppression of the Impact of Full-Scale Wind-Power Converters on Subsynchronous Damping," IEEE Systems Journal, vol. 7, pp. 700-712, dec 2013.

[2] Y. Wu, W. Yang, X. Xiao, J. Zhang, and C. Luo, "Analysis of frequently over-threshold subsynchronous oscillation and its suppression by subsynchronous oscillation dynamic suppressor," IET Generation, Transmission \& Distribution, vol. 10, pp. 2127-2137, jun 2016.

[3] B. Kroposki, B. Johnson, Y. Zhang, V. Gevorgian, P. Denholm, B.-M. Hodge, and B. Hannegan, "Achieving a $100 \%$ Renewable Grid: Operating Electric Power Systems with Extremely High Levels of Variable Renewable Energy," IEEE Power and Energy Magazine, vol. 15, pp. 6173, mar 2017.

[4] H. Zhou, J. Chen, Q. Liu, Y. Hu, and J. Zhu, "Sub-synchronous torsional interaction with VSC-HVDC affected by feed-forward compensations in current controllers," The Journal of Engineering, vol. 2017, pp. 2140 2145, jan 2017.

[5] Y. C. Choo, A. P. Agalgaonkar, K. M. Muttaqi, S. Perera, and M. Negnevitsky, "Analysis of subsynchronous torsional interaction of HVDC system integrated hydro units with small generator-to-turbine inertia ratios," IEEE Transactions on Power Systems, vol. 29, pp. 1064-1076, may 2014. 
[6] R. Thirumalaivasan, M. Janaki, and N. Prabhu, "Damping of SSR Using Subsynchronous Current Suppressor With SSSC," IEEE Transactions on Power Systems, vol. 28, pp. 64-74, feb 2013.

[7] T. Kwasnik, B. O. Sigrin, and D. A. Bielen, "Quantifying Resolution Implications for Agent-based Distributed Energy Resource Customer Adoption Models," tech. rep., National Renewable Energy Laboratory (NREL), Golden, CO (United States), jan 2019.

[8] L. Harnefors, "Analysis of Subsynchronous Torsional Interaction With Power Electronic Converters," IEEE Transactions on Power Systems, vol. 22, pp. 305-313, feb 2007.

[9] L. Harnefors, X. Wang, A. G. Yepes, and F. Blaabjerg, "PassivityBased Stability Assessment of Grid-Connected VSCs-An Overview," IEEE Journal of Emerging and Selected Topics in Power Electronics, vol. 4, pp. 116-125, mar 2016.

[10] K. M. Alawasa, Y. A. R. I. Mohamed, and W. Xu, "Active mitigation of subsynchronous interactions between PWM voltage-source converters and power networks," IEEE Transactions on Power Electronics, vol. 29, no. 1, pp. 121-134, 2014.

[11] "First benchmark model for computer simulation of subsynchronous resonance," IEEE Transactions on Power Apparatus and Systems, vol. 96, pp. 1565-1572, sep 1977.

[12] P. M. P. M. Anderson, B. L. B. L. Agrawal, J. E. J. E. Van Ness, and IEEE Power Engineering Society., Subsynchronous resonance in power systems. IEEE Press, 1990.

[13] R. K. Varma and R. Salehi, "SSR Mitigation With a New Control of PV Solar Farm as STATCOM (PV-STATCOM)," IEEE Transactions on Sustainable Energy, vol. 8, pp. 1473-1483, oct 2017.

[14] "IEEE Standard for Interconnection and Interoperability of Distributed Energy Resources with Associated Electric Power Systems Interfaces." 2018.

[15] A. F. Hoke, A. A. Nelson, J. Tan, R. Mahmud, V. Gevorgian, M. Elkhatib, C. Antonio, D. Arakawa, and K. Fong, "The FrequencyWatt Function: Simulation and Testing for the Hawaiian Electric Companies," tech. rep., National Renewable Energy Laboratory (NREL), Golden, CO (United States), jul 2017. 